\title{
Non-Destructive quality assessment of bio-engineering parts using Industrial Micro X-ray Computed Tomography: A Review
}

\author{
Jitendra Singh Rathore ${ }^{\mathrm{a},}$, Prateek Saxena ${ }^{\mathrm{b}, \mathrm{c}}$ \\ ${ }^{a}$ CEA-LIST, Département d'Imagerie, Simulation et Contrôle, 91191 Gif-sur-Yvette, France \\ ${ }^{b}$ Sustainable Manufacturing Systems Centre, School of Aerospace, Transport and Manufacturing, \\ Cranfield University, Bedfordshire, UK \\ ${ }^{c}$ School of Engineering, Indian Institute of Technology Mandi, Mandi, Himachal Pradesh, \\ 175005 India
}

\begin{abstract}
Bio-engineering parts are widely used in hearing aid applications, dental applications, inhalation therapy blends, etc. Conventional tactile measurement techniques offer a limitation in the quality assessment of such parts due to the complex shape of the part and also due to their inability to measure internal features in a threedimensional space. Industrial micro X-ray computed tomography ( $\mu \mathrm{XCT})$ is a robust characterization technique designed to maintain part quality, facilitate precision manufacturing, and reduce material waste due to part rejection. This work highlights the limitations and challenges of conventional measurement techniques in the assessment of the quality of bio-engineering components. Capabilities of $\mu \mathrm{XCT}$ are presented and its application in the bio-engineering sector is discussed in this work.
\end{abstract}

Keywords: Micro X-ray Computed Tomography, Bio-engineering, Quality control, Non Destructive Technique, Precision manufacturing

\section{Introduction}

Bio-engineering components are conventionally manufactured with micro injection molding e.g. micro filters for acoustics, hearing aids, implantable clips,

Email addresses: jitendra-singh.rathore@hotmail.com (Jitendra Singh Rathore), pra.9000@gmail.com (Prateek Saxena)

Preprint submitted to Materials Letters February 19, 2021 
etc. In recent times, additive manufacturing is also being utilized especially for the manufacturing of metallic parts such as implant solutions and scaffolds. Bioengineering parts are characterized by small and complex features often with dimensions in the order of microns which also require very high precision to ensure effective usage. For example, accurate volume measurement of cavities is required for predicting the maximum dosage that can be loaded on a drug-eluting stent [1]. Depending on the quality criteria, several techniques are being used conventionally, such as optical and/or probe-based instruments are utilized for dimensional measurements, the Archimedes method for total porosity, and the gravimetric method for volume/wear measurements. However, they pose specific limitations for parts with intrinsic features and complex shapes. Hansen et al. [2] showed a classification of instruments with respect to the aspect ratio of the measuring parts, which is a crucial criterion to define the complexity of the features to be measured. In addition, there are several challenges and/or limitations associated with most measuring instruments, which further justify the need for new technologies. For examples, probe-based systems could not access the complex features and optical instrument are not fully three-dimensional (3D) as well as probe-based machines could induce unwanted damages and/or deformations (in polymeric components) due to clamping and probing forces leading to a large measurement uncertainty [3]. Industrial Micro X-ray Computed Tomography $(\mu \mathrm{XCT})$ could provide a solution for such complex bio-engineering parts for their holistic measurements both internally and externally. The unique feature of this measurement technique is that it is entirely three-dimensional, which enables holistic data acquisition.

\section{Industrial X-ray CT}

$\mu \mathrm{XCT}$ is an X-ray 3D imaging technology which is based on the same principle as in the medical CT systems but could utilize high power and achieve very high resolution for industrial use [6,7]. Over the years, it has become an established technique for non-destructive quality control in industrial environments across various domains $[8,9,10,11]$. This Non-Destructive Technique (NDT) offers great advantages such as, in particular, no sampling preparation, complete 3D information, no sectioning, etc. In contrast to the tactile coordinate measuring machines (CMMs), a CT scan of an object acquires all surface points of the part including all hidden features which otherwise remain inaccessible non-destructively with other methods. Fig. 1a shows the working principle of $\mu \mathrm{XCT}$ systems, the $\mathrm{X}$-ray projections are acquired for a complete rotation cycle. The acquired projec- 


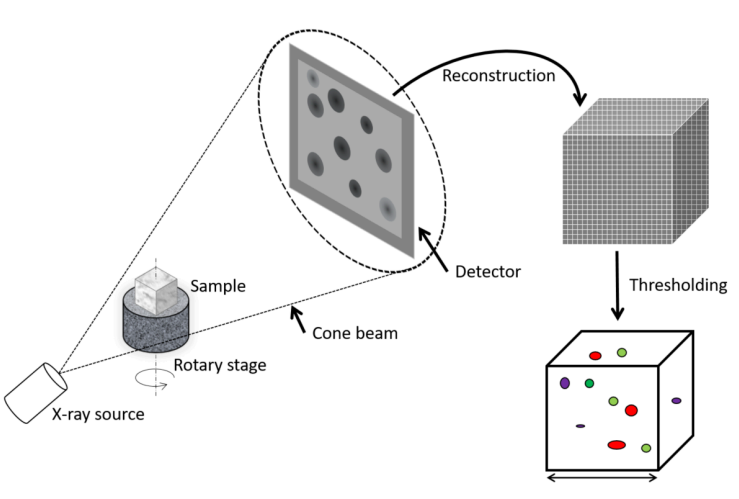

(a)

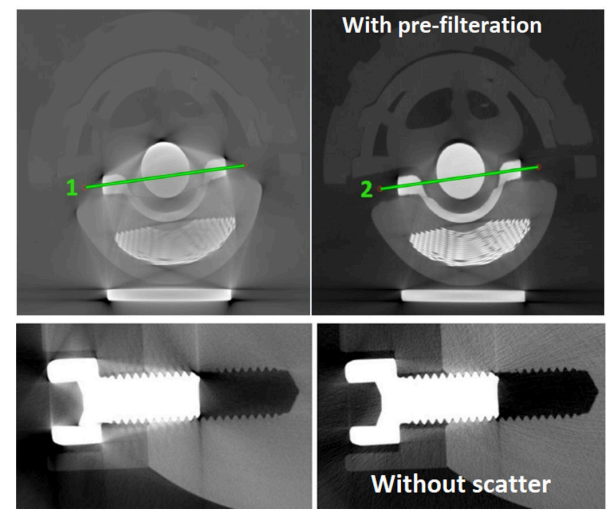

(b)

Figure 1: (a) Working principle of $\mu \mathrm{XCT}$ and (b) typical beam hardening artifact (top) and corresponding minimization with pre-filteration adopted from [4] and typical scattering artifacts adopted from [5]

tions are post-processed and a volumetric dataset is obtained, which is composed of gray-level-based voxels (three-dimensional pixels); smaller voxel size represents a higher resolution of the CT data. Then, a thresholding step is required which defines the boundary between the material and the background; an accurate threshold ensures precise quantitative analyses.

Although, $\mu \mathrm{XCT}$ has no limitations related to the probe access, because of the high penetration power of X-rays. However, there exist several limitations that affect the quality of the acquired data. According to the German guideline, VDI/VDE 2630 - 1.2 [12], the influencing factors can be categorized into five groups of parameters: system, workpiece, data processing, environment, and operator. It is out of the scope of this review to discuss the influencing factors in detail, nevertheless, it is recommended to refer $[4,5]$ for further details. The quality of acquisition is affected in form of one or more artifacts, most common are the beam hardening and scattering artifacts which arise due to the polychromatic nature of X-ray beam and scattering of the X-ray photons after interaction with material respectively. Some of the artifacts can be avoided to some extent with pre-scanning strategies, moreover, the development of software based corrections is a major interest of researchers. An example of typical beam hardening and scattering artifacts is presented in Fig. 1b

$\mu \mathrm{XCT}$ can be utilized for various metrological tasks ranging from evaluating the global deviations against the nominal geometry to quantifying the local internal defects [23, 24] as well as fiber orientation in composites [7, 10, 25]. Numer- 
Table 1: Common bio-engineering applications where $\mu \mathrm{XCT}$ is utilized for quality assessment

\begin{tabular}{llll}
\hline Application & Material & Quality criterion & Reference \\
\hline Acetabular cup (metallic) & - & Surface texture & {$[13]$} \\
Artificial Urinary Sphincter & AMS 800 & Wall thickness and & {$[14]$} \\
Microfludics & (Boston Scientific, Boston, USA) & Model Validation & \\
Dental file & Cyclic Olefin Copolymers (COC) & Shrinkage and warpage & {$[15]$} \\
Drug-eluting stent & Ti-Ni alloy & Dimensional & {$[16]$} \\
Hearing aid toggle & - & Volume measurement & {$[1]$} \\
Hip joint prostheses & Liquid crystal polymer (LCP) & Dimensional & {$[17]$} \\
Inhalation therapy blends & Polyethylene & Wear & {$[3,18]$} \\
& $\alpha$-lactose monohydrate powders & Powder characterization & {$[19]$} \\
Biomaterials & Polycarbonate (PC) & & \\
Scaffolds & - & Morphological characterization & {$[20,21]$} \\
\hline
\end{tabular}

ous studies have reported the use of $\mu \mathrm{XCT}$ for quality inspection in the context of the bio-engineering components. Table 1 discusses some common applications highlighting the quality criteria. It shows that $\mu \mathrm{XCT}$ can be used for a wide range of materials and qualitative and quantitative analyses based on several quality aspects related to the functional performance of the part; which makes $\mu \mathrm{XCT}$ a very versatile technique for quality assessment. We have broadly categorized the various measurement tasks among external and internal quality assessments.

\subsection{External quality assessment}

There could be several external quality criteria which are defined for efficient functional performance of a specific part. Some of the important criteria have been highlighted and discussed in detail.

\subsubsection{Dimensional measurements}

For a manufacturer, dimensional accuracy is the primary objective, which becomes extremely important when the part is characterized by strict tolerances. As mentioned, bio-engineering parts usually consist of miniature functional features with strict tolerances. Some typical measurands are height/depth in microfluidics, internal/external diameters in hearing aid toggles, etc. Fig. 2a shows an example of a hearing aid toggle as produced and the measurands defining the functional performance of such toggles are outer diameter $(D)$, inner diameter $(d)$ and pillar height $(H)$ (Fig. 2b) [17]. The results obtained from $\mu \mathrm{XCT}$ for three such toggles are compared against the reference measurements taken with an optical coordinate measuring machine (OCMM) and the deviations are plotted in Fig. 2c. In general, 
the deviations are as low as $0 \mu m$ but always $\leq 10 \mu m$, which depicts the ability of the $\mu \mathrm{XCT}$ to make measurements comparable to the conventional OCMM.

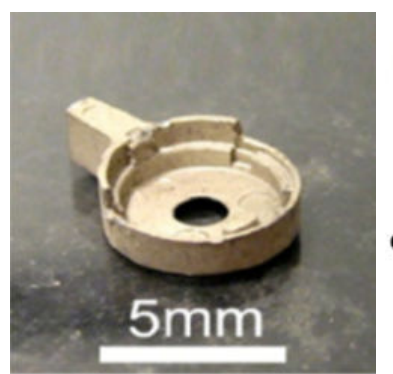

(a)

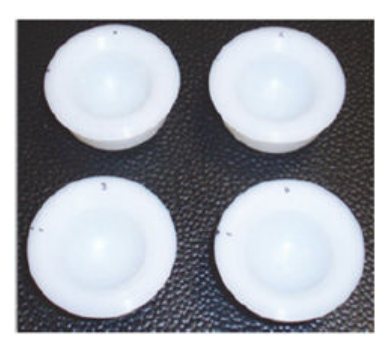

(d)

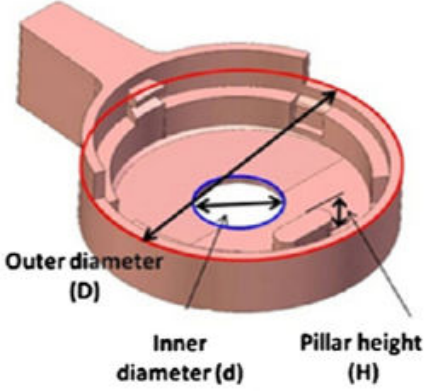

(b)

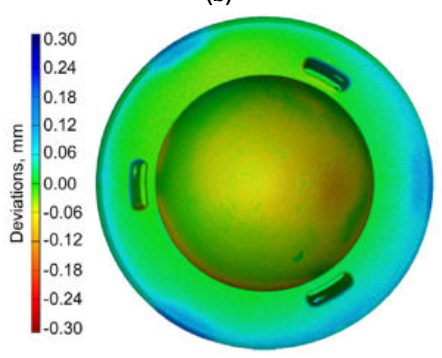

(e)

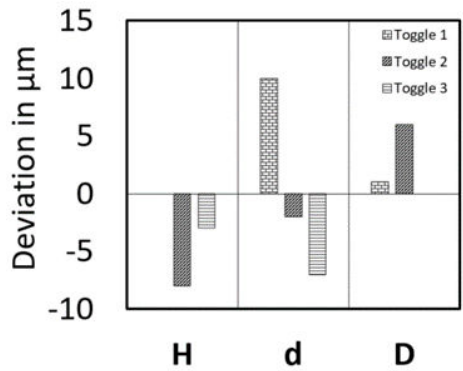

(c)

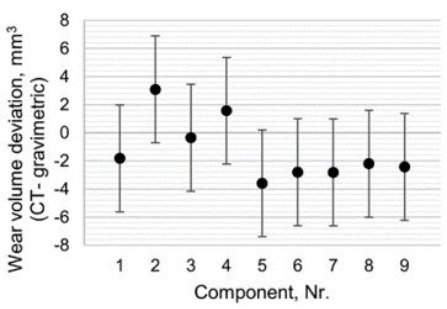

(f)

Figure 2: Examples of dimensional and wear measurements by $\mu \mathrm{XCT}$ : (a) Hearing aid toggle as produced, (b) Defined quality measurands, (c) Obtained CT measurements (deviations from reference values) [16], (d) Polyethylene acetabular cups with $30 \mathrm{~mm}$ diameter of spherical bearing surface [26], (e) Wear/deformation map of acetabular components and (f) Deviations between volumetric wear measurements obtained by CT and gravimetric method [3].

\subsubsection{Wear measurements}

Measurement of wear volumes and geometric characterization are of primary importance for understanding wear mechanisms and improving technological solutions provided by prosthetic joint components manufacturers. The laboratory determination of wear rate is an important part of the pre-clinical validation of prostheses [26]. The most commonly applied method for wear evaluation of prosthetic joint components is the gravimetric method. It is generally accurate and reliable but has got certain limitations. In particular, gravimetric measurements cannot provide information about the localization of wear damage and deformation over the component's surface. As an alternative, tactile coordinate measuring machines are used to evaluate both volumetric wears and wear damage distribution over the worn surface. However, considering polymeric prosthetic compo- 
nents, these machines could induce unwanted damages and/or deformations due to clamping and probing forces leading to a large measurement uncertainty [3]. In such a case, $\mu \mathrm{XCT}$ can be used for the measurement of volumetric wear as well as for the determination of the local distribution of wear and deformation without any risk of further damaging or deforming polymer components.

An example of a hip prosthesis with ultra-high molecular weight polyethylene (UHMWPE) cups is shown in Fig. 2d. Geometrical measurements were conducted before and after the wear test using $\mu \mathrm{XCT}$ to be able to evaluate the volumetric wear. A deviation map obtained from the CT data showing local wear and deformations as distributed over the worn surfaces is shown in Fig. 2e. The difference between CT volumetric wear measurements and the conventional gravimetric method is reported in Fig. 2f. The results indicate that there is not a clear systematic trend of differences between CT and gravimetric results. However, the majority of the measured deviations are negative and this might be caused by the presence of systematic errors [3]. Nevertheless, this study further exploited CT capabilities.

\subsubsection{Nominal-actual comparison}

One of the most important applications of micro injection molding is microfluidics, which demands holistic quality control for mass replication. $\mu \mathrm{XCT}$ as an emerging technology provides a significant improvement in quality control and reverse engineering due to its non-destructive nature. Rathore et al. [15] showcased the use of $\mu \mathrm{XCT}$ for such applications, the authors used a specific microfluidic system which was manufactured in two different materials: Cyclic Olefin Copolymers (COC) and Polycarbonate (PC). $\mu \mathrm{XCT}$ provided significantly important results as far as the part quality is concerned. The $\mu \mathrm{XCT}$ based measurement of the overall linear shrinkage of part revealed that the shrinkage is $x$ and $y$ directions are lower than $0.5 \%$ for COC and slightly higher than $0.5 \%$ for PC. In $z$ direction, the COC part exhibits $3 \%$ shrinkage whereas PC exhibits $3.5 \%$. Therefore, it can also be concluded that the part with PC witnesses more shrinkage compared to the COC. The non-uniform shrinkage resulted in warpage of the part, which was also possible to quantify from the CT results using the nominal-actual comparison.

\subsubsection{Wall-thickness analysis}

Wall-thickness analysis is also one of the important quality parameters, which needs to be quantified in a number of bio-engineering applications. An important example is the Artificial Urinary Sphincter (AUS) device used for surgical treatment of urinary incontinence as demonstrated in [14]. An AUS cuff is a fun- 
damental component of the device, providing the mechanical action addressed to urethral occlusion and therefore, it needs to be investigated by computational approach. $\mu \mathrm{XCT}$ is used to acquire the cuff conformation both in deflated and inflated conditions, for an accurate definition of the geometrical characteristics. An accurate 3D geometrical model of the cuff in the deflated configuration is obtained using $\mu \mathrm{XCT}$ (Fig. 3a) and the wall-thickness and internal distance between surfaces (Fig. 3b). Furthermore, model validation is achieved by comparing the measurements provided by the tomography of the AUS cuff inflated at selected pressure levels and the corresponding numerical results. The relative error was reported to be below $2.5 \%$. Thanks to $\mu \mathrm{XCT}$, this study is the first step in the understanding of AUS mechanical behavior and enables the development of computational tools for the analysis of the lumen occlusion process.

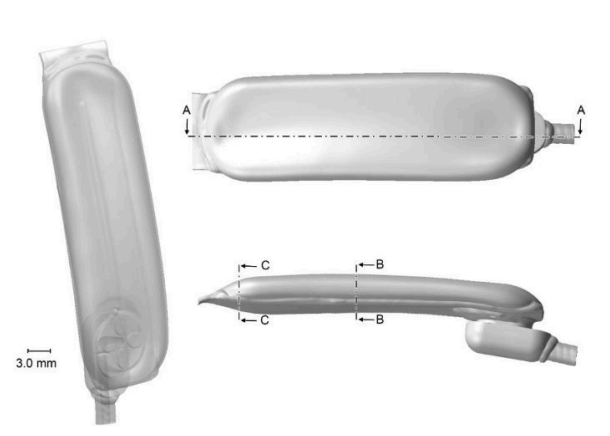

(a)

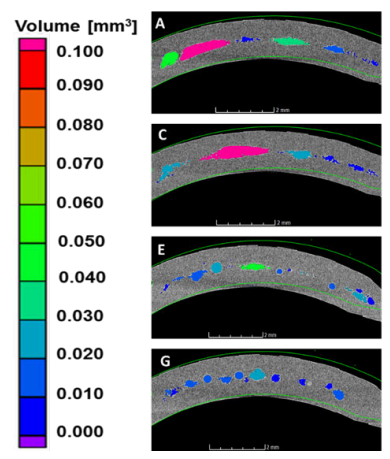

(c)

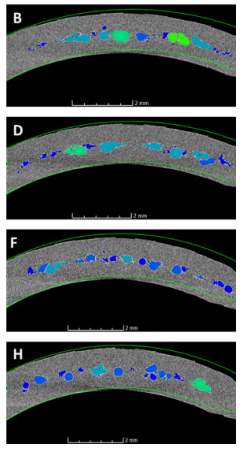

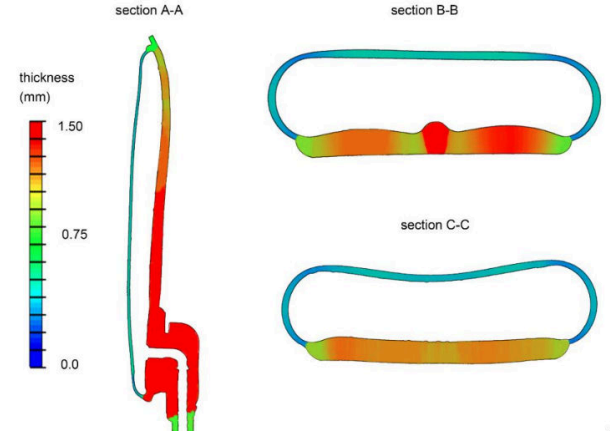

(b)

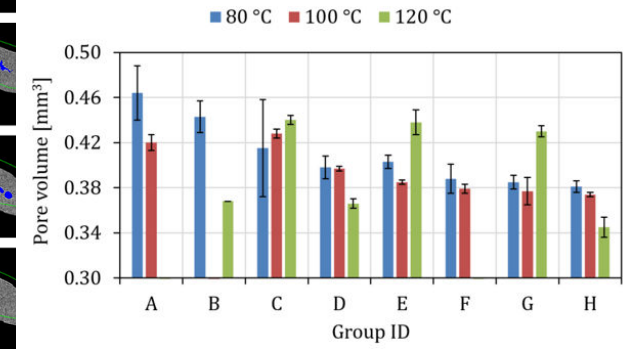

(d)

Figure 3: (a) Reconstructed CT volume of AUS device with different views of the geometrical model of the cuff with indicated sections $A-A$, B-B and $C$ - $C$, (b) Contour of the material wallthickness at the corresponding sections [14], (c) $\mu$ XCT Measurement results of total pore volume and (d) Cross sectional view showing the pore morphology at different process conditions which are represented with letters $A$ to $H$ [24]. 


\subsection{Internal quality assessment}

Depending on the manufacturing process several parts are suffered from poor internal quality mainly with a presence of pore or inclusions. X-ray tomography can be used for inspection of internal flaws which is presented with some case studies.

\subsubsection{Porosity}

Internal porosity or defect is a very important issue in several manufacturing processes, particularly injection molding, where the molten polymer solidifies in the cavity, and therefore accurate detection of such porosity is of great importance for optimizing process settings. $\mu \mathrm{XCT}$ enables the user to obtain holistic information about the internal porosity including the 3D spatial distribution of the pores and the local and global pore volumes which otherwise may not be solely possible with other conventional methods. Rathore et al. [24] studied a micro-component with a V-shaped geometry which is present in several medical applications, especially where the hinging function is required e.g. ligating clips. They used a design-of-experiment approach concerning the process parameters (denoted with letter $A$ to $H$ ) at three mold temperatures $80^{\circ} \mathrm{C}, 100^{\circ} \mathrm{C}$ and $120^{\circ} \mathrm{C}$ and used CT based feedback for correlation. The cross-sections obtained from the CT reconstruction are shown in Fig. 3c at mold temperature $80^{\circ} \mathrm{C}$. The variation of pore sizes can be seen with varying process conditions. Furthermore, the quantified mean pore volumes with the standard deviation are plotted for all the process condition at different mold temperatures (see Fig. 3d). As it can be seen with color-coding, the process settings $A$ at $80^{\circ} \mathrm{C}$ yields the maximum void volume while $H$ at $120^{\circ} \mathrm{C}$ yields the minimum void volume. Moreover, the pores are distributed mainly along the central symmetrical plane (parting plane) of the part and individual pore volume ranges from $0.010 \mathrm{~mm}^{3}$ to $0.10 \mathrm{~mm}^{3}$. This pattern of pore distribution can be attributed to the fact that the material around the central plane is the last to solidify and, therefore, it is more prone to air/gas entrapment and/or differential shrinkage.

\subsubsection{Characterization of scaffolds and biomaterials}

Biomaterials and scaffolds that are used as bone substitutes to repair critical defects serve mainly as a combined mechanical support and osteoregeneration functions and differ in their biological properties, such as osteoconduction, osteoinduction, and osteogenesis, as well as in their osteointegration and structural support $[27,28]$. 
The use of additive manufacturing for scaffold application has become a preferred choice thanks to the NDT through $\mu \mathrm{XCT}$. It can provide complete quality control of the AM scaffolds which allows producing scaffolds with desired microarchitecture, texture analysis, different porosities, strut sizes, and spacing [29]. The use of $\mu \mathrm{XCT}$ has been demonstrated for evaluating surface texture [13], dimensional accuracy of uniform and gradient scaffolds $[22,30]$ as well as scaffolds with intricate geometries (i.e. diamond lattice, triply periodic minimal surfaces) that enhance cell migration while retaining a high degree of mechanical and structural rigidity $[31,32]$. Complex scaffold structures produced by electrospinning can be investigated using $\mu \mathrm{XCT}$ (see Fig. 4a), allowing for more complex measurements such as fiber distribution and orientation [33]. Furthermore, in-situ $\mu \mathrm{XCT}$ has also been used to characterize the compressive failure of 3D printed ceramic scaffolds for bone regeneration, enabling a deeper insight between the structural and failure behavior to further tailor the $3 \mathrm{D}$ printing process for ceramic biomaterials Fig. $\mathbf{4 b}$.

In very recent work, Gajjar et al. [19] presented the use of $\mu \mathrm{XCT}$ for the characterization of dry powder inhalers (DPIs) which consist of a powder mixture of active pharmaceutical ingredients (API) and inert carriers (excipients) contained in a device that deagglomerates the particles upon inhalation to deliver a dose to the lung. Such DPI mixtures are required to be accurately characterized for their particle size distributions, surface roughness, fines' contents, and flow properties. Understanding the microstructure of the powder formulation is crucial, yet current characterization methods such as laser diffraction (LD) and optical microscopy (OM) are not sufficient. Therefore, $\mu$ XCT was utilized for characterizing the DPI formulations and the numerical results were also compared with the laser diffraction. $\mu \mathrm{XCT}$ based 3D visualisations of Lactohale 100 (4894 particles) and Lactohale 200 (308554 particles) can be seen in Fig. 4c and Fig. 4d respectively. The difference between the sieving and milling processes is visible with Lactohale 200 showing a higher number of intrinsic lactose fines than Lactohale 100. The size distribution plot of the number of fines is shown in Fig. 4e in comparison with plot obtained from LD. Both Lactohale 100 and Lactohale 200 have main peaks around $100 \mu \mathrm{m}$ corresponding to the coarse lactose, with Lactohale 200 showing a second smaller peak around $20 \mu \mathrm{m}$ corresponding to lactose fines. Furthermore, the calculated mean densities of lactose fines as $9426 \pm 559$ particles per $\mathrm{mm}^{3}$ and $66458 \pm 6033$ particles per $\mathrm{mm}^{3}$ for Lactohale 100 and Lactohale 200 respectively. 


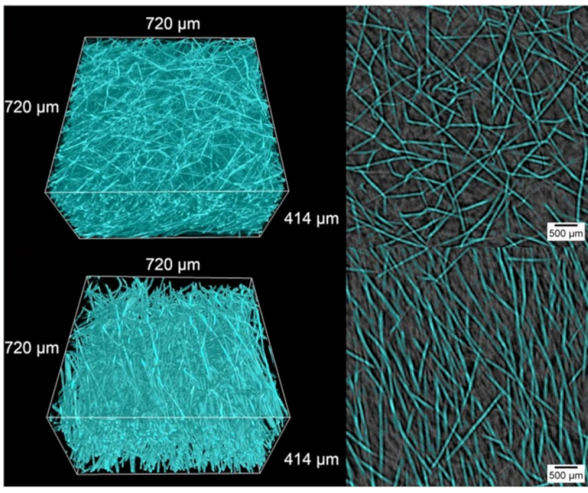

(a)

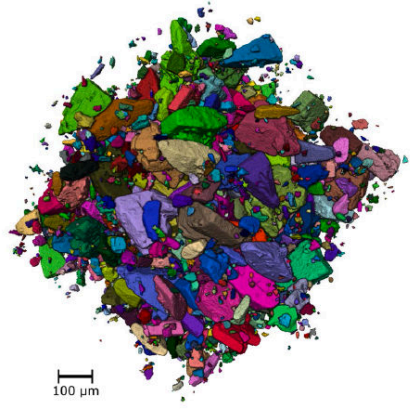

(c)
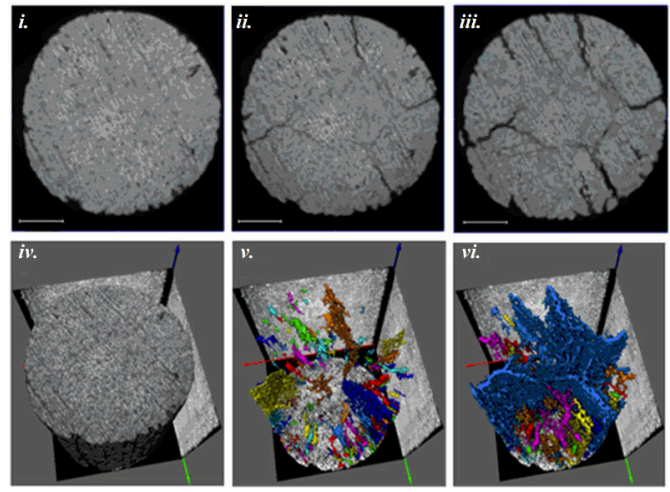

(b)

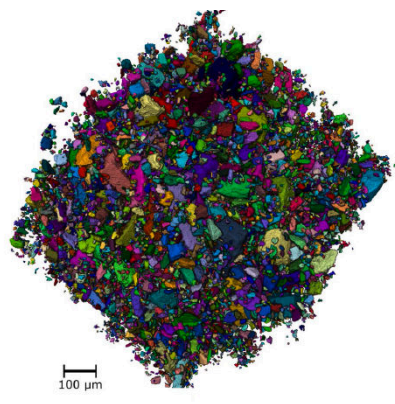

(d)

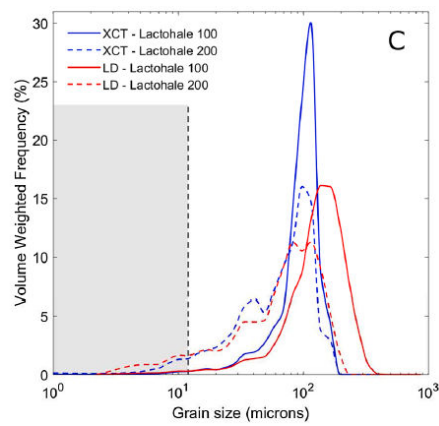

(e)

Figure 4: (a) Visualisation of polymer PCL scaffolds: randomly oriented (top) and well-aligned structures (bottom) manufactured using an electro-spinning process (3D view in left column and transverse section through sample height in right column) [33], (b) In-situ failure of a 3D powder printed ceramic scaffold under intermittent compression: representative CT cross-sections corresponding to the initial state $(i$.$) , the progression of the crack generation (ii.) and the fractured$ state (iii.) and the corresponding reconstruction showing the mode of crack (coloured) generation and propagation in the bulk scaffold ( (iv)-(vi) ) [22], (c) CT visualisation of Lactohale 100, (d) Lactohale 200 (with one colour per particle) and (e) the size distributions for the two grades from $\mathrm{CT}$ and LD with fine lactose of size $<12 \mathrm{~m}$ highlighted in shaded grey region [19].

\section{Limitations and outlook}

As highlighted in section 2, $\mu \mathrm{XCT}$ does have some limitations. Some of the recent works have studied these limitations e.g. the influence of resolution on measurement results has been studied by Rathore et al. [34]. In addition, recent research has reported several solution for beam hardening correction [35] and scattering correction [36, 37]. Moreover, one of the most important limitations for $\mu \mathrm{XCT}$ based measurements is the lack of international standards to guide uncertainty estimation which is attributed to these numerous influencing factors and 
their interactions [12]. Hence, there is an evident need for future research on various influences and their quantification which could eventually lead to standardization. Despite all these limitations, $\mu \mathrm{XCT}$ has a great potential to become the most preferred metrology solution in industrial environments especially with the increasing use of additive manufacturing parts.

\section{Conclusions}

Bio-engineering components typically require precision manufacturing and are primarily manufactured from injection molding or additive manufacturing techniques. The quality control of such parts is rather difficult with the use of tactile and optical measurement techniques due to the complex geometry of the parts and the internal micro-features. The utility and application of the industrial $\mu \mathrm{XCT}$ for the quality assessment of the bio-engineering components is presented in this work. This non-destructive quality tool can be used for precision manufacturing, wear measurement, wall thickness analysis, identification of internal porosity, deviation from the geometry of the intended part, and for the characterization of scaffolds and biomaterials. As a result, industrial $\mu \mathrm{XCT}$ scanners have the potential to reduce material waste due to part rejection, thus improving the production throughput of the manufacturing unit.

\section{References}

[1] S. Carmignato, E. Savio, Traceable volume measurements using coordinate measuring systems, CIRP annals 60 (2011) 519-522.

[2] H. N. Hansen, K. Carneiro, H. Haitjema, L. De Chiffre, Dimensional micro and nano metrology, CIRP annals 55 (2006) 721-743.

[3] F. Zanini, S. Carmignato, E. Savio, S. Affatato, Uncertainty determination for X-ray computed tomography wear assessment of polyethylene hip joint prostheses, Precision Engineering 52 (2018) 477-483.

[4] H. Villarraga-Gómez, E. L. Herazo, S. T. Smith, X-ray computed tomography: from medical imaging to dimensional metrology, Precision Engineering 60 (2019) 544-569.

[5] A. Stolfi, L. De Chiffre, S. Kasperl, Error sources, in: Industrial X-Ray Computed Tomography, Springer, 2018, pp. 143-184. 
[6] P. Hermanek, J. S. Rathore, V. Aloisi, S. Carmignato, Principles of xray computed tomography, in: Industrial X-Ray Computed Tomography, Springer, 2018, pp. 25-67.

[7] J. P. Kruth, M. Bartscher, S. Carmignato, R. Schmitt, L. De Chiffre, A. Weckenmann, Computed tomography for dimensional metrology, CIRP annals 60 (2011) 821-842.

[8] P. Saxena, G. Bissacco, F. J. Bedka, A. Stolfi, Tooling for production of the green fiber bottle, Procedia CIRP 69 (2018) 348 - 353. 25th CIRP Life Cycle Engineering (LCE) Conference, 30 April - 2 May 2018, Copenhagen, Denmark.

[9] P. Saxena, G. Bissacco, K. Ælkær Meinert, A. H. Danielak, M. M. Ribó, D. B. Pedersen, Soft tooling process chain for the manufacturing of microfunctional features on molds used for molding of paper bottles, Journal of Manufacturing Processes 54 (2020) 129 - 137.

[10] J. S. Rathore, T. Konopczyński, J. Hesser, G. Lucchetta, S. Carmignato, Investigation on tomographic-based nondestructive characterization of short glass fiber-reinforced composites as obtained from micro injection molding, Journal of Nondestructive Evaluation, Diagnostics and Prognostics of Engineering Systems 3 (2020).

[11] P. Saxena, G. Bissacco, A. Stolfi, L. De Chiffre, Characterizing green fiber bottle prototypes using computed tomography, in: 7th Conference on Industrial Computed Tomography (iCT 2017), 2017.

[12] V. D. Ingenieure, Vdi/vde 2630 blatt 1.2: Computertomografie in der dimensionellen messtechnik. einflussgrößen auf das messergebnis und empfehlungen für dimensionelle computertomografie-messungen (2008) 1-15.

[13] S. Lou, L. Pagani, W. Zeng, M. U. Ghori, X. Jiang, P. J. Scott, Surface texture evaluation of additively manufactured metallic cellular scaffolds for acetabular implants using x-ray computed tomography, Bio-Design and Manufacturing 2 (2019) 55-64.

[14] A. N. Natali, C. G. Fontanella, S. Todros, P. G. Pavan, S. Carmignato, F. Zanini, E. L. Carniel, Conformation and mechanics of the polymeric cuff of artificial urinary sphincter, Mathematical Biosciences and Engineering 17 (2020) 3894. 
[15] J. S. Rathore, S. Carmignato, G. Lucchetta, Improving ct quality control of a micro injection molded part by multisensor data fusion, in: Proceedings of the 6th Conference on Industrial Computed Tomography, Wels, Austria, 2016, pp. 9-12.

[16] J. Yagüe-Fabra, G. Tosello, S. Ontiveros, R. Jiménez, J. Rasmussen, Dimensional quality control of ti-ni dental file by optical coordinate metrology and computed tomography, in: 14th International Conference of the European Society for Precision Engineering and Nanotechnology, euspen, 2014, pp. 102-105.

[17] S. Ontiveros, J. Yagüe-Fabra, R. Jiménez, G. Tosello, S. Gasparin, A. Pierobon, S. Carmignato, H. N. Hansen, Dimensional measurement of micromoulded parts by computed tomography, Measurement Science and Technology 23 (2012) 125401.

[18] S. Affatato, F. Zanini, S. Carmignato, Micro x-ray computed tomography mass loss assessment of different uhmwpe: A hip joint simulator study on standard vs. cross-linked polyethylene, PLoS One 12 (2017) e0170263.

[19] P. Gajjar, I. Styliari, T. Nguyen, J. Carr, X. Chen, J. Elliott, R. Hammond, T. Burnett, K. Roberts, P. Withers, et al., 3d characterisation of dry powder inhaler formulations: Developing x-ray micro computed tomography approaches, European Journal of Pharmaceutics and Biopharmaceutics (2020).

[20] M. P. Fernandez, F. Witte, G. Tozzi, Applications of x-ray computed tomography for the evaluation of biomaterial-mediated bone regeneration in critical-sized defects, Journal of Microscopy 277 (2020) 179-196.

[21] B. Arbez, J.-D. Kün-Darbois, T. Convert, B. Guillaume, P. Mercier, L. Hubert, D. Chappard, Biomaterial granules used for filling bone defects constitute 3d scaffolds: porosity, microarchitecture and molecular composition analyzed by microct and raman microspectroscopy, Journal of Biomedical Materials Research Part B: Applied Biomaterials 107 (2019) 415-423.

[22] S. Mandal, S. Meininger, U. Gbureck, B. Basu, 3d powder printed tetracalcium phosphate scaffold with phytic acid binder: Fabrication, microstructure and in situ x-ray tomography analysis of compressive failure, Journal of Materials Science: Materials in Medicine 29 (2018) 29. 
[23] N. E. Gorji, P. Saxena, M. Corfield, A. Clare, J.-P. Rueff, J. Bogan, P. G. González, M. Snelgrove, G. Hughes, R. O’Connor, R. Raghavendra, D. Brabazon, A new method for assessing the recyclability of powders within powder bed fusion process, Materials Characterization 161 (2020) 110167.

[24] J. S. Rathore, G. Lucchetta, S. Carmignato, Towards optimization of $\mu$ injection molding process for a new $\mathrm{v}$-shaped geometrical component using $\mathrm{x}$-ray ct-based quality characterization, Journal of Manufacturing and Materials Processing 3 (2019) 13.

[25] P. Saxena, G. Bissacco, C. Gundlach, V. A. Dahl, C. H. Trinderup, A. B. Dahl, Process characterization for molding of paper bottles using computed tomography and structure tensor analysis, E-Journal of Nondestructive Testing \& Ultrasonics 24 (3) (2019).

[26] S. Carmignato, M. Balcon, F. Zanini, Investigation on the accuracy of ct measurements for wear testing of prosthetic joint components, in: Proceedings of the Conference on Industrial Computed Tomography (ICT), 2014.

[27] L. Roseti, V. Parisi, M. Petretta, C. Cavallo, G. Desando, I. Bartolotti, B. Grigolo, Scaffolds for bone tissue engineering: state of the art and new perspectives, Materials Science and Engineering: C 78 (2017) 1246-1262.

[28] W. Wang, K. W. Yeung, Bone grafts and biomaterials substitutes for bone defect repair: A review, Bioactive materials 2 (2017) 224-247.

[29] X. Zhang, Y. Zhang, Tissue engineering applications of three-dimensional bioprinting, Cell biochemistry and biophysics 72 (2015) 777-782.

[30] S. M. Bittner, B. T. Smith, L. Diaz-Gomez, C. D. Hudgins, A. J. Melchiorri, D. W. Scott, J. P. Fisher, A. G. Mikos, Fabrication and mechanical characterization of $3 \mathrm{~d}$ printed vertical uniform and gradient scaffolds for bone and osteochondral tissue engineering, Acta biomaterialia 90 (2019) 37-48.

[31] N. J. Castro, J. O'brien, L. G. Zhang, Integrating biologically inspired nanomaterials and table-top stereolithography for $3 \mathrm{~d}$ printed biomimetic osteochondral scaffolds, Nanoscale 7 (2015) 14010-14022. 
[32] Y. Li, J. Zhou, P. Pavanram, M. Leeflang, L. Fockaert, B. Pouran, N. Tümer, K.-U. Schröder, J. Mol, H. Weinans, et al., Additively manufactured biodegradable porous magnesium, Acta biomaterialia 67 (2018) 378-392.

[33] S. Shkarina, R. Shkarin, V. Weinhardt, E. Melnik, G. Vacun, P. J. Kluger, K. Loza, M. Epple, S. I. Ivlev, T. Baumbach, et al., 3d biodegradable scaffolds of polycaprolactone with silicate-containing hydroxyapatite microparticles for bone tissue engineering: High-resolution tomography and in vitro study, Scientific reports 8 (2018) 1-13.

[34] J. S. Rathore, C. Vienne, Y. Quinsat, C. Tournier, Influence of resolution on the x-ray ct-based measurements of metallic am lattice structures, Weld World 64 (2020) 1367-1376.

[35] W. Cao, Development of beam hardening correction algorithms for industrial computed tomography (2019).

[36] M. Krenkel, M. Erler, S. Tzoumas, C. Kuhn, X-ray scatter removal for artifact free ct imaging, in: Proceedings of the Conference on Industrial Computed Tomography (ICT), 2020.

[37] X. Xia, X. Hu, Z. Han, D. Zhang, Y. Xu, J. Zou, Scatter correction in cone beam ct for metal additive manufacturing components, Jom 71 (2019) 10821087. 
Non-destructive quality assessment of bio-engineering parts using Industrial Micro X-ray Computed Tomography: A review

Rathore, Jitendra Singh

Elsevier

Rathore JS, Saxena P. (2021) Non-destructive quality assessment of bio-engineering parts using Industrial Micro X-ray Computed Tomography: A review. Materials Letters, Volume 287, March 2021, Article number 129252

https://doi.org/10.1016/j.matlet.2020.129252

Downloaded from Cranfield Library Services E-Repository 